\title{
Effect of Gradual Demineralization on the Mineral Fraction and Mechanical Properties of Cortical Bone *
}

\author{
Masahiro TODOH ${ }^{* *}$, Shigeru TADANO**, Bijay GIRI ${ }^{* *}$ \\ and Masahiro NISHIMOTO** \\ **Division of Human Mechanical Systems and Design, Graduate School of Engineering, Hokkaido \\ University, \\ Kita-13, Nishi-8, Kita-ku, Sapporo, Japan \\ E-mail: todoh@eng.hokudai.ac.jp
}

\begin{abstract}
Bone is often regarded as a composite material consisting of hydroxyapatite (HAp-like) mineral particles, organic matrix (mostly Type I collagen) and water phases in microscopic scale. The mechanical properties of bone at macroscopic scale depend on the structural organization and properties of constituents in the microscopic scale. In the attempts of understanding the effect of microscopic constituent on the mechanical properties of bone, the relationship between mechanical properties and mineral content of intact or completely demineralized samples have been studied. Even a slight alteration in the mineral content would have significant effect on the mechanical properties. In this work, the effect of degree of demineralization to the mechanical properties of bovine cortical bone was examined by gradually removing the mineral content and measuring constituents at every step till almost no traces of minerals were observed. Specimens were demineralized in 10\% disodium EDTA solutions for 12, 24, 48, 72 hours and 14 days. The volume fraction of each structural constituent in the demineralized specimens was calculated from their X-ray absorption characteristics and quantifying transmitted X-ray intensity. Tensile tests were performed to measure the elastic modulus and ultimate strength of the demineralized specimens. This work shows the strong dependence of elastic modulus and ultimate strength of cortical bone to the mineral content as microscopic constituent. The degree of dependence with mineral loss has been demonstrated precisely so to provide the importance of mineral content and its role on the mechanical functioning of bone.
\end{abstract}

Key words: Biomechanics, Bone, Mineral Content, Mechanical Properties, X-Ray Absorption, Demineralization

\section{Introduction}

Bone has a hierarchical structure at different structural levels and is often regarded as a composite material consisting of hydroxyapatite (HAp-like) mineral particles, organic matrix (mostly Type I collagen) and water phases in microscopic scale. The mechanical properties of bone at macroscopic scale depend on the structural organization and properties of constituents in the microscopic scale ${ }^{(1)-(4)}$. Considerable attempts have been made to investigate the effect of the microscopic constituent on the mechanical properties of bone. It is the interaction between the mineral and organic material that determines the mechanical properties. The effect of mineral content on the stiffness, strength or viscoelasticity of bone has been widely investigated ${ }^{(5)-(12)}$. However, most of these studies discuss the relationship

${ }^{*}$ Received 25 Nov., 2008 (No. 08-0823) [DOI: 10.1299/jbse.4.230] 
between mechanical properties and mineral content of intact or completely demineralized samples. Even a slight alteration in the mineral content would have significant effect on the mechanical properties. One of the major factors contributing to increased osteoporosis fracture risk with age is decreasing mineral content ${ }^{(13)}$. Experimental analysis on the gradual demineralization of bone and thereby effect on the mechanical properties is limited.

The current study examined the effect of degree of demineralization to the mechanical properties of bovine cortical bone by gradually removing the mineral content and measuring constituents at every step till almost no traces of minerals were observed. Specimens were demineralized in 10\% disodium EDTA solutions for 12, 24, 48, 72 hours and 14 days. The volume fraction of each structural constituent in the demineralized specimens was calculated from their X-ray absorption characteristics and quantifying transmitted X-ray intensity. Tensile tests were performed to measure the elastic modulus and ultimate strength of the demineralized specimens.

\section{Materials and methods}

\subsection{Demineralization}

Thirty cortical bone specimens with an average size of $50.0 \times 10.0 \times 1.0 \mathrm{~mm}$ were harvested from the mid-diaphyses of three bovine femurs (approximately 2-years old). The specimens were arranged with their longer sides bone-axially and their breadth circumferentially. Rough cuts were done by using a hand-saw and fine cuts by using a low-speed diamond saw (SBT650, South Bay Technology, Inc. USA). Five specimens were gradually demineralized by immersing in $10 \%$ disodium EDTA (ethylene diamine tetraacetic acid) solutions $\left(4^{\circ} \mathrm{C}, \mathrm{pH}=7.4\right)$ in each duration $(12,24,48,72$ hours and 14 days). The specimen after 14 days was confirmed to be totally demineralized from an X-ray diffraction image, where traces of existence of HAp were not observed.

Each specimen after demineralization was immediately weighed in air with its adherent film of water by using an electronic balance (GF-200, A\&D Corp. Ltd., Japan). They were further weighed after drying in vacuum oven (AVO-250, AS ONE Corp., Japan) at room temperature $\left(23^{\circ} \mathrm{C}\right)$ for 4 hours. Water content was calculated from the difference of mass of the specimen before and after drying.

\subsection{Volume fraction}

The mineral phase in bone composite has higher X-ray absorbance than organic matrix. Volume fraction of each structural constituent (minerals and collagen matrix) was determined from their X-ray absorption characteristics. Characteristic (monochromatic) X-ray (Mo-K $\alpha$ ) was generated from X-ray diffractometer (RINT2000, Rigaku, Co. Japan). The intensity transmitted from the specimen focused with $0.5 \mathrm{~mm}$ diameter collimator was detected using the scintillation counter. The transmitted X-ray intensity decreases exponentially with thickness given as

$$
I=I_{0} \exp \left(-\mu_{\text {bone }} \cdot t\right)
$$

where $I_{0}$ is the incident X-ray intensity, $t$ is thickness of specimen, $\mu_{\text {bone }}$ is the linear absorption coefficient of bone.

The volume fraction of each constituent was calculated from the following relation.

$$
\left[\begin{array}{cc}
\mu_{\text {hap }} & \mu_{\text {col }} \\
1 & 1
\end{array}\right]\left[\begin{array}{l}
v_{\text {hap }} \\
v_{\text {col }}
\end{array}\right]=\left[\begin{array}{l}
\mu_{\text {bone }} \\
v_{\text {bone }}
\end{array}\right]
$$

where $\mu_{\text {bone }}$ and $v_{\text {bone }}$ are the linear absorption coefficient and volume fraction of bone, $\mu_{\text {hap }}$ and $v_{\text {hap }}$ are the linear absorption coefficient and volume fraction of HAp, $\mu_{c o l}$ and $v_{c o l}$ are the linear absorption coefficient and volume fraction of collagen constituent. 
Furthermore, the specimen consisting of number of constituents has the following relation for the linear absorption coefficient $\mu$,

$$
\frac{\mu}{\rho}=\sum g_{i}\left(\frac{\mu}{\rho}\right)_{i}
$$

where $\rho$ is mean density of the specimen, $g_{i}$ is ratio of weight of a constituent to the total weight of the specimen, and $(\mu / \rho)_{i}$ is mass absorption coefficient.

Here, the linear absorption coefficients of hydroxyapatite $\left(\mathrm{HAp}: \mathrm{Ca}_{10}\left(\mathrm{PO}_{4}\right)_{6}(\mathrm{OH})_{2}\right)$ and Type I collagen molecule (glycine: $\mathrm{C}_{2} \mathrm{H}_{5} \mathrm{NO}_{2}$; proline: $\mathrm{C}_{5} \mathrm{H}_{9} \mathrm{NO}_{2}$; hydroxyproline: $\mathrm{C}_{2} \mathrm{H}_{5} \mathrm{NO}_{2}$ ) were considered. The linear absorption coefficients were determined from the elemental values given in Table $1^{(14)}$. The mean densities of HAp $\left(\rho_{\text {hap }}\right)$ and collagen $\left(\rho_{\text {col }}\right)$ are $3.16^{(15)}$ and $1.41\left(\mathrm{~g} / \mathrm{cm}^{3}\right){ }^{(16)}$ respectively. Calculated linear absorption coefficients $\mu_{\text {hap }}$ and $\mu_{c o l}$ are $29.4 / \mathrm{cm}$ and $1.24 / \mathrm{cm}$ respectively for Mo-K $\alpha$ (wave length $\lambda=0.07107 \mathrm{~nm}$ ) used in this experiment.

Table 1 Mass absorption coefficient of the elements for characteristic X-ray Mo-K ${ }^{(14)}$

\begin{tabular}{cccc}
\hline Wave length & $\mathrm{nm}$ & Absorber & Mass absorption coefficients $\mu / \rho \mathrm{m}^{2} / \mathrm{g}$ \\
\hline & $\mathrm{Ca}$ & 1.83 & \\
0.07107 & $\mathrm{P}$ & 0.789 & \\
$(\mathrm{Mo}-\mathrm{K} \alpha)$ & $\mathrm{O}$ & 0.131 & \\
& $\mathrm{~N}$ & 0.092 & \\
& $\mathrm{C}$ & 0.063 & \\
& $\mathrm{H}$ & 0.038 \\
\hline
\end{tabular}

\subsection{Mechanical tests}

Tensile tests were performed using a universal testing machine (Model4411, Instron Corp., USA) to evaluate the mechanical properties of cortical specimens with different demineralized duration. Both ends of cortical specimens were fixed to the heads of the universal testing machine binding by two metal plates by two screws. The strain of each cortical specimen was estimated from the distance of both heads, because it was confirmed that the fixation has no slip by preliminary test by using the strain gauge. All the experiments for demineralized samples were carried out at constant strain rate of 0.02 $\mathrm{mm} / \mathrm{min}$ in a saline bath at room temperature. The elastic modulus and ultimate strength for each specimen were then obtained. The elastic modulus was then calculated from the linear region $\left(\varepsilon<2000 \times 10^{-6}\right)$ of stress-strain curve of each specimen. The ultimate strength was determined as the maximal stress under tensile loading.

\section{Results}

The change in mass and volume $(n=5)$ of the specimen with the immersion duration in EDTA solution are shown in Fig. 1a and 1b, respectively. The mass in both dry and wet conditions decrease with increasing immersion duration. The dry volume decrease with increasing immersion duration, but the wet volume remains constant with demineralization duration. This is because the free space in the tissue due to loss of mineral crystals (mean density $\left.\rho_{\text {hap }}=3.16 \mathrm{~g} / \mathrm{cm}^{3}\right)^{(15)}$ during demineralization had been occupied by the water, and hence the overall volume remains unchanged.

The trend of decrease of transmitted X-ray fraction $\left(I / I_{0}\right)$ having different absorption coefficients at different demineralized duration are plotted for different penetration depth of the specimen (Fig. 2). The linear absorption coefficient of specimen after 2 weeks immersion is $1.30 / \mathrm{cm}(\mathrm{n}=4)$, which is close to the theoretical value for organic collagen $(1.24 / \mathrm{cm})$. The linear absorption coefficient of intact specimen is found to be $14.8 / \mathrm{cm}$ 
$(n=4)$. The linear absorption coefficients for other specimens at different immersion durations decrease with increasing demineralization.
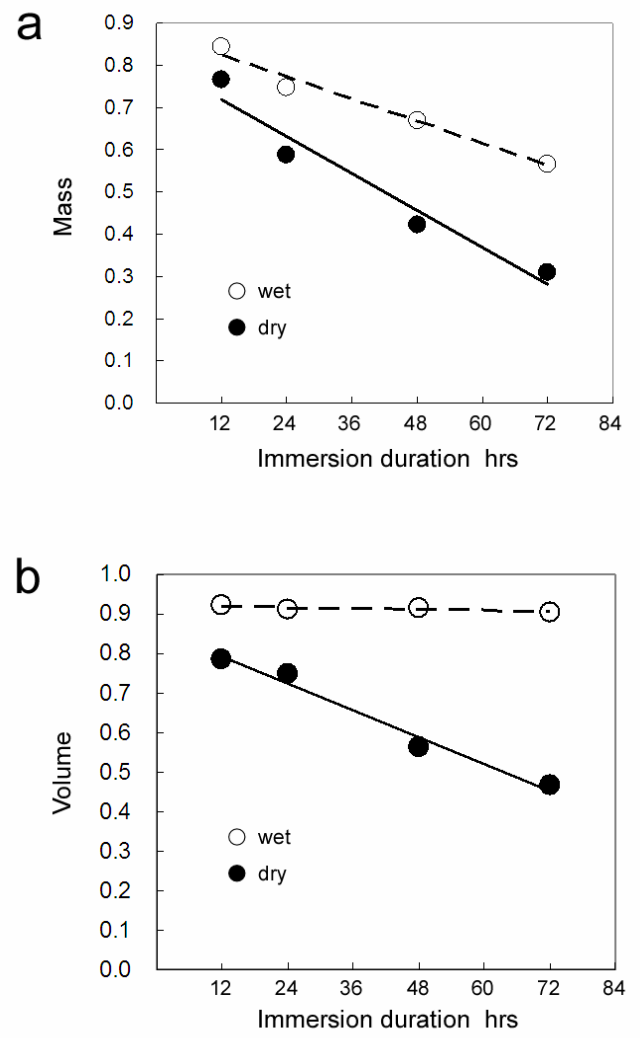

Fig. 1 Changes in mass (a) and volume (b) of the specimen in both dry and wet conditions with the demineralized duration.

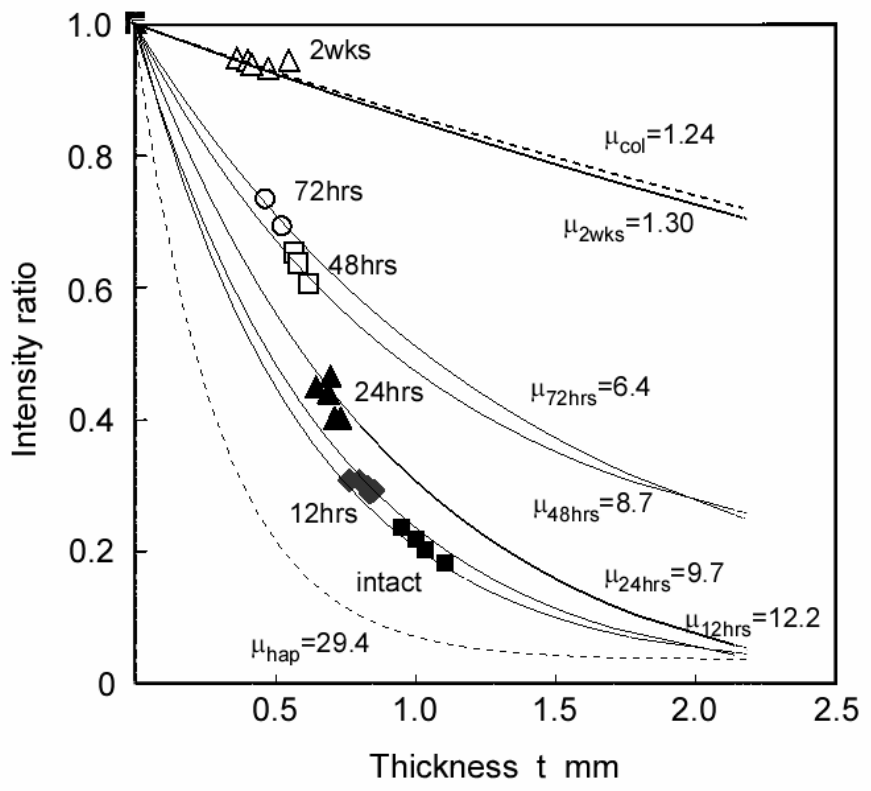

Fig. 2 Transmitted X-ray fraction $I / I_{0}$ at different demineralized duration. The linear absorption coefficients were calculated by approximating the relationships between the transmitted X-ray fraction and thickness of cortical bone specimen. 
Figure 3 shows the volume fraction of each structural constituent, namely HAp, collagen and water, obtained after different immersion duration. The volume fractions of HAp and collagen were obtained from the transmitted fraction X-ray intensity. Water content was obtained from the difference of mass after drying the specimen. The intact cortical specimen contains almost same fractions of HAp and collagen (HAp: $46.5 \%$, collagen: $45.4 \%$ ) and the rest was water $(8.1 \%)$. As demineralization proceeds, the volume fraction of HAp also decreases gradually and the negligible value is observed at 2 weeks immersion period. A slight decrease in collagen matrix is observed. The volume of decreased mineral content was balanced by water, which can be observed with increasing water content with increasing demineralization (also according to Fig. 1).

The typical tensile stress-strain curves for demineralized tissues at different immersion duration are shown in Fig. 4. The slopes of initial linear region of the curves decrease with increasing immersion duration. The ultimate strain of demineralized specimen for 2 weeks is very large compared to the others.

The elastic modulus (obtained from the linear region of Fig. 4) and tensile strength (obtained from the maximum stress) vary exponentially with the increase of mineral volume fraction as shown in Fig. 5a and 5 b respectively. The elastic modulus of intact specimen is $6.5 \mathrm{GPa}$, whereas that of completely demineralized specimen ( 2 weeks immersion) is much less, equal to $0.066 \mathrm{GPa}$. The tensile strength of intact tissue is $77 \mathrm{MPa}$ and that of completely demineralized tissue is $12 \mathrm{MPa}$.

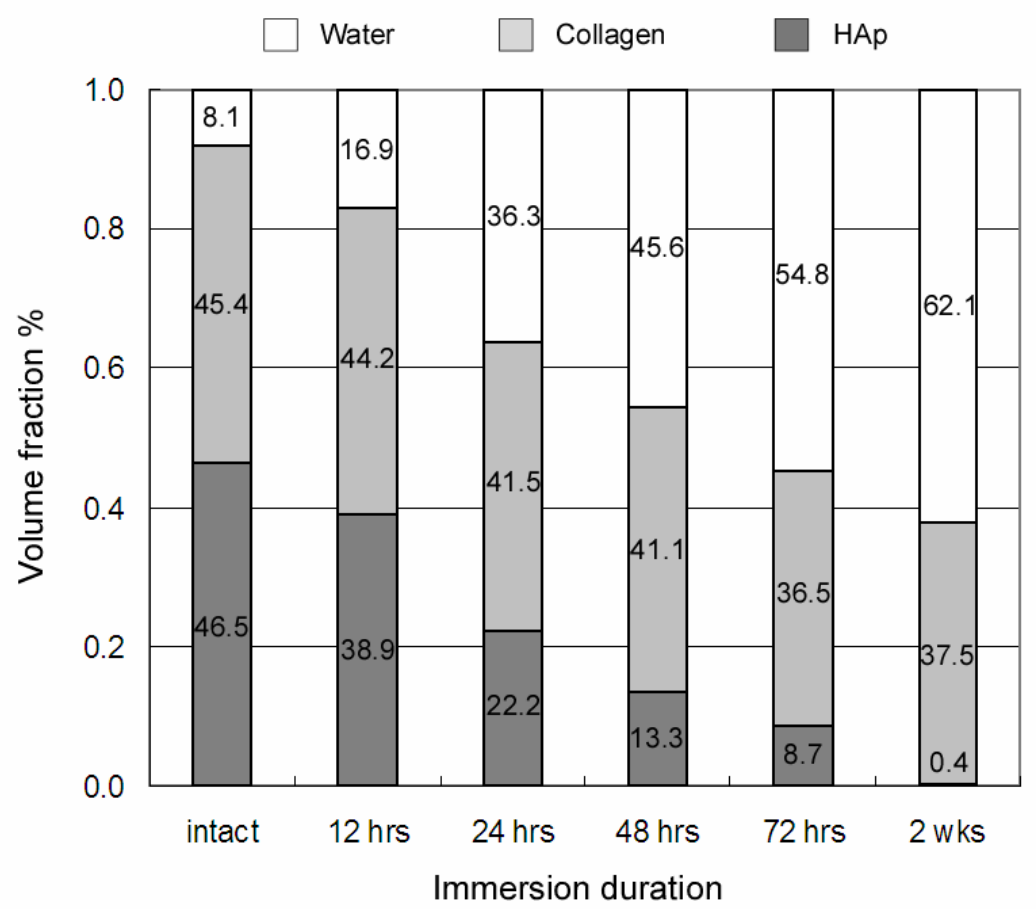

Fig. 3 Changes in volume fraction of each structural constituents (HAp, collagen and water) after gradual demineralization. The volume fraction of each structural constituent in the specimens was calculated from $\mathrm{X}$-ray absorption characteristics and quantifying transmitted X-ray intensity. 


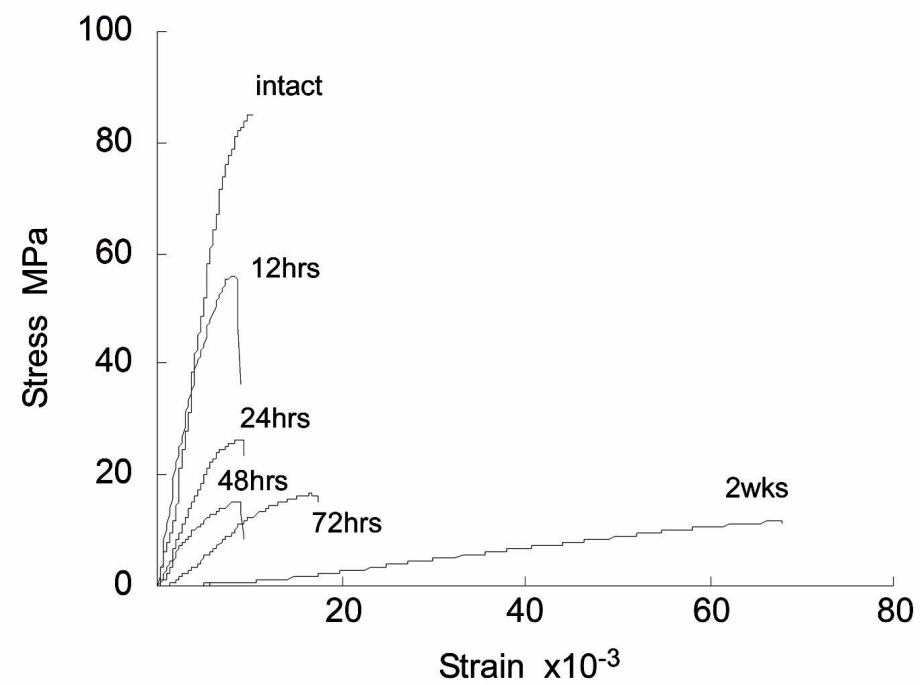

Fig. 4 Stress-strain curves for demineralized specimens at different immersion duration. The elastic moduli are obtained from the linear region of the curves and tensile strength are obtained from the maximum stress.

a

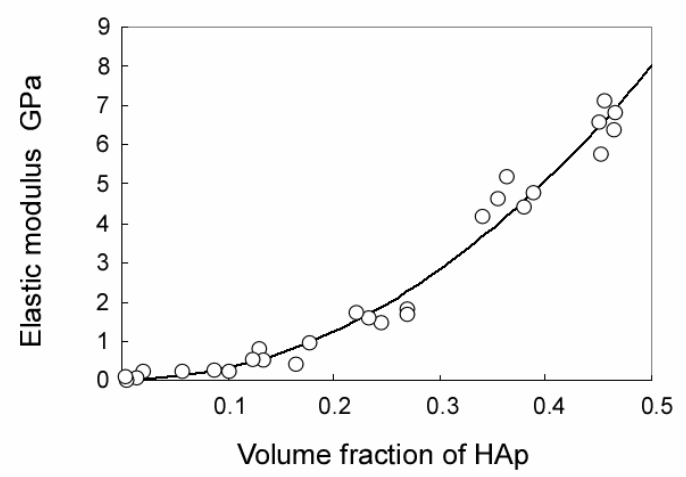

b

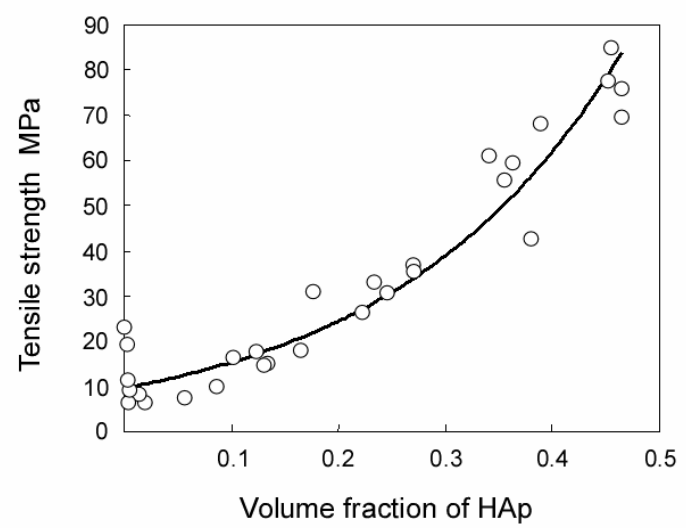

Fig. 5 Changes in elastic modulus (a) and tensile strength (b) by gradually eliminating minerals from bone. 


\section{Discussion}

This study reveals the mechanical characteristics of gradually demineralized cortical bone samples at short intervals unlike the attempts with demineralized tissue in the existing literatures, and hence the effect of gradual loss of mineral content on bone has been demonstrated in precise scale. Disodium EDTA solution, which has been demonstrated to preserve the biological architecture of collagen matrix ${ }^{(17)(18)}$, was used as demineralizing agent for all the specimens. Non-destructive and non-invasive X-ray absorption procedure was applied to estimate the volume fraction of demineralized samples. In the case of the partial EDTA treatment, the decrease of mineralized volume fraction were greatest in the outer regions of the specimen ${ }^{(5)}$, thereby affecting the tensile properties measured in this study less than flexural properties.

With the gradual loss of minerals, the water content in the specimen was found to increase (Fig. 3), and hence higher absorbance compared to collagen matrix (theoretical value of $\mu$ for HAp is $29.4 / \mathrm{cm}$ and for collageneous molecule is $1.24 / \mathrm{cm})^{(19)}$, linear absorption coefficients of the specimens are also observed to decrease gradually according to different demineralization duration (Fig. 2) with the loss of minerals (Fig. 3). After 2 weeks' immersion, X-ray diffraction showed there was no apatite crystals, where no traces of diffraction from the crystals was observed, which could also be verified from respective linear absorption coefficient value $(1.30 / \mathrm{cm})$ close to that of theoretical value of collagen. The calculated volume fraction at this instant also showed negligible traces of minerals (i.e. $0.3 \%$ ) (Fig. 3).

The tensile tests performed on bovine cortical specimens show gradual decrease in strength with the immersion duration (Fig. 4). Compared to previously reported elastic modulus values for completely demineralized specimens $\left(750 \mathrm{MPa}{ }^{(6)}, 370 \mathrm{MPa}{ }^{(12)}, 613\right.$ $\left.\mathrm{MPa}{ }^{(20)}\right)$, we obtained much lower value $(66 \mathrm{MPa})$. Similarly the ultimate strength compared to previous reports $\left(39.5 \mathrm{MPa}{ }^{(6)}, 34 \mathrm{MPa}{ }^{(11)}\right.$ and $\left.61.5 \mathrm{MPa}{ }^{(20)}\right)$ is also low (12 $\mathrm{MPa}$ ) as obtained in this work. Here, the tensile tests were performed with the specimen immersed in saline to produce a uniform environment. All the above cited previous studies performed the mechanical tests by periodically moistening the specimen with saline at room-temperature. The loss of moisture at room temperature increases the strength and stiffness; stiffness of collagen increases ${ }^{(19)(21)}$, and the molecular diameter of collagen decreases ${ }^{(22)}$ with the decrease in hydration.

The previous studies ${ }^{(7)(10)}$ obtained the exponential relationship of elastic modulus with the mineral content (from about 60 to $70 \%$ ) from the individual difference of intact cortical bone specimen. The exponential relationship of elastic modulus with the mineral content (from 0.4 to $46.5 \%$ ) also obtained from the incompletely demineralized cortical bone in this work. The mineral crystals in bone can be regarded as rigid particles and the collagen fibers as matrix. The mineral phase is supposed to contribute to bone stiffness, whereas the characteristics of collagen matrix predominantly determine the toughness of bone ${ }^{(6)}$. Hence, the increase of ultimate strain with the increasing demineralization duration obtained in this work also shows agreement with this. Under the simplest composite law (e.g. $E_{\text {bone }}=$ $\left.E_{\text {hap }} v_{\text {hap }}+E_{\text {col }} v_{c o l}\right)$ in the case of homogenious particle-dispersed composite material, the mechanical properties were linear to the mineral content. The nonlinearity of relation between volume fraction of HAp and mechanical properties obtained in this study shows the dependence on the anisotropic arrangement of HAp crystals and collagen matrix, and the interface of both constituents. To clarify the mechanical composite model of cortical bone at microscopic level, the precise structural observation of HAp crystals and collagen matrix and the measurement of the mechanical behavior of both constituents experimentally were necessary.

In the existing literatures related to demineralization using EDTA solution, the concentration of calcium in the solution or mineral density of heat treated specimen to 
eliminate all the organic constituents, are considered to quantify the mineral loss, however, such approaches are limited to in vitro quantification. In this work, the volume fraction of each structural constituent (mineral phase and collagen matrix) was quantified from X-ray absorption characteristics. The non-destructive and non-invasive measurement merit of $\mathrm{X}$-rays has led to the extensive application of X-rays in the study of mineralized tissue, which is also considered to provide the outcomes at precise level.

By gradually eliminating minerals from the bone and reveals corresponding mechanical properties at short intervals, the current work shows the strong dependence of elastic modulus and ultimate strength of cortical bone to the mineral content as microscopic constituent. The degree of dependence with mineral loss has been demonstrated precisely so to provide the importance of mineral content and its role on the mechanical functioning of bone.

\section{Acknowledgement}

This research was partially supported by Grant-in Aid for Young Scientists (B) (No. 18760069) from the Ministry of Education, Culture, Sports, Science and Technology of Japan.

\section{References}

(1) Fratzl P., Gupta H. S., Paschalis E. P. and Roschger P., Structure and Mechanical Quality of the Collagen-Mineral Nano-Composite in Bone, Journal of Materials Chemistry, Vol. 14, No. 14 (2004), pp. 2115-2123.

(2) Ji B. and Gao H., Elastic Properties of Nanocomposite Structure of Bone, Composites Science and Technology, Vol. 66, No. 9 (2006), pp. 1212-1218.

(3) Rho J. Y., Kuhn-Spearing L. and Zioupos P., Mechanical Properties and the Hierarchical Structure of Bone, Medical Engineering \& Physics, Vol. 20, No. 2 (1998), pp. 92-102.

(4) Yamashita J., Li X., Furman B. R., Rawls H. R., Wang X. and Agrawal C. M., Collagen and Bone Viscoelasticity: A Dynamic Mechanical Analysis, Journal of Biomedical Materials Research, Vol. 63, No. 1 (2002), pp. 31-36.

(5) Broz J. J., Simske S. J. and Greenberg A. R., Material and Compositional Properties of Selectively Demineralized Cortical Bone, Journal of Biomechanics, Vol. 28, No. 11 (1995), pp. 1357-1368.

(6) Burstein A. H., Zika J. M., Heiple K. G. and Klein L, Contribution of Collagen and Mineral to the Elastic-Plastic Properties of Bone, The Journal of Bone \& Joint Surgery, Vol. 57A, No. 7 (1975), pp. 956-961.

(7) Currey J. D., The Mechanical Consequences of Variation in the Mineral Content of Bone. Journal of Biomechanics, Vol. 2, No. 1 (1969), pp. 1-11.

(8) Currey J. D., The Effects of Strain Rate, Reconstruction and Mineral Content on Some Mechanical Properties of Bovine Bone, Journal of Biomechanics, Vol. 8, No. 1 (1975), pp. 81-86.

(9) Jonas J., Burns J., Abel E. W., Cresswell M. J., Strain J. J. and Peterson C. R., A Technique for the Tensile Testing of Demineralized Bone, Journal of Biomechanics, Vol. 26, No. 3 (1993), pp. 271-276.

(10) Vose G. P. and Kubala A. L., Bone Strength - Its Relationship to X-Ray Determined Ash Content, Human Biology, Vol. 31 (1959), pp. 262-270.

(11) Wang T. and Feng Z., Dynamic Mechanical Properties of Cortical Bone: The Effect of Mineral Content, Materials Letters, Vol. 59, No. 18 (2005), pp. 2277-2280.

(12) Wright T. M., Vosburgh F. and Burstein A. H., Permanent Deformation of Compact Bone Monitored by Acoustic Emission, Journal of Biomechanics, Vol. 14, No. 6 (1981), pp. 
405-409.

(13) Roschger P., Paschalis E. P., Fratzl P. and Klaushofer K., Bone Mineralization Density Distribution in Health and Disease, Bone, Vol. 42, No. 3 (2008), pp. 456-466.

(14) Victoreen, J. A., The Calculation of X-Ray Mass Absorption Coefficients, Journal of Applied Physics, Vol. 20 (1949), pp. 1141-1147.

(15) Joint Committee on Powder Diffraction Standards, Powder Diffraction File, 9-432.

(16) Lees S., Water Content in Type I Collagen Tissues Calculated from the Generalized Packing Model, International Journal of Biological Macromolecule, Vol. 8 (1986), pp. 66-72.

(17) Hasegawa K., Turner C. H. and Burr D. B., Contribution of Collagen and Mineral to the Elastic Anisotropy of Bone, Calcified Tissue International, Vol. 55, No. 5 (1994), pp. 381-386.

(18) Kivitanta I., Tammi M., Lappalainen R., Kussela T. and Helminen H. J., The Rate of Calcium Extraction during EDTA Decalcification from Thin Bone Slices as Assessed with Atomic Absorption Spectrophotometry, Histochemistry, Vol. 68 (1980), pp. 119-127.

(19) Nomura S., Hiltner A., Lando J. B. and Baer E., Interaction of Water with Native Collagen, Biopolymers, Vol. 16, No. 2 (1977), pp. 231-246.

(20) Bowman S. M., Zeind J., Gibson L. J., Hayes W. C. and McMahon T. A., The Tensile Behavior of Demineralized Bovine Cortical Bone, Journal of Biomechanics, Vol. 29, No. 11 (1996), pp. 1497-1501.

(21) Pineri M. H, Escoubes M. and Roche G., Water-Collagen Interactions: Calorimetric and Mechanical Experiments, Biopolymers, Vol. 17, No. 12 (1978), pp. 2799-2815.

(22) Lees S., A Mixed Packing Model for Bone Collagen, Calcified Tissue Intertnational, Vol. 33, No. 1 (1981), pp. 591-602. 